\title{
Co-trimoxazole sensitivity testing: comparison of separate and combined disk agar diffusion techniques
}

\author{
SGB AMYES AND WA TELFER BRUNTON \\ From the Department of Bacteriology, The Medical School, University of Edinburgh, \\ Teviot Place, Edinburgh EH8 9AG, UK
}

SUMMARY Five hundred and seven strains of bacteria isolated from the urine of patients with significant bacteriuria (more than $10^{8}$ colony-forming units per litre) were tested for sensitivity to co-trimoxazole by the agar diffusion technique. Each organism was tested with a combined disk containing trimethoprim and sulphamethoxazole in a primary sensitivity test and, at a standardised inoculum, with both a combined disk and separate disks of trimethoprim and sulphamethoxazole. The results show that combined disk testing does not always indicate the sensitivity patterns of the organisms being tested.

Since its introduction in 1968, co-trimoxazole has become widely used for the treatment of respiratory, gastrointestinal, and urinary tract infections. ${ }^{1}$ Despite the popularity of this preparation, it is a matter of continuing debate whether synergism between the components, trimethoprim and sulphamethoxazole, is of importance in vivo. ${ }^{2} 3$

In the laboratory, sensitivity testing of cotrimoxazole has been the subject of much controversy. Some workers believe that synergy may be important and insist that sensitivity testing by the agar diffusion technique should be performed with separate disks so that synergy can be demonstrated.

On the other hand, primary single disk testing, with an inoculum that has not been standardised, is more convenient and produces a more rapid result. However, by this method information cannot be gained about the sensitivity of the test organism to the individual components of co-trimoxazole or about the occurrence of synergy.

In this study we examine the performance of a single combined disk in a primary sensitivity test. The combined disk contained trimethoprim and sulphamethoxazole in a ratio of $1: 20$, as is used in some routine laboratories. These results are compared with those obtained in tests with separate disks of trimethoprim and sulphamethoxazole and a standardised inoculum; in the latter tests, the potency of the sulphonamide disk was amended to take account of the antagonism between the two drugs on laboratory media as described by Amyes. ${ }^{4}$

Accepted for publication 14 July 1980

\section{Material and methods}

Specimens of urine were submitted in sterile $30 \mathrm{ml}$ plastic containers (Sterilin, Middx, UK) from patients under the care of clinicians in the Royal Infirmary of Edinburgh or of local general practitioners. A maximum of four hours was permitted between voiding of the sample and its processing in the laboratory.

The specimens were cultured by the blotting paper-strip method on to MacConkey Agar (Oxoid CM7B, Basingstoke, Hants, UK), ${ }^{5}$ and the remaining urine was centrifuged at $3000 \mathrm{rpm}$ for 5 minutes. A loopful of the deposit was used to prepare a Gram-stained smear. If microscopic examination of the smear indicated that significant bacteriuria was likely to be confirmed, a loopful of deposit was used to seed a plate containing Diagnostic Sensitivity Test Agar (DST, Oxoid), ${ }^{5}$ and a 'combined' disk containing $1 \mu \mathrm{g}$ trimethoprim and $20 \mu \mathrm{g}$ sulphamethoxazole (Mast Laboratories, Liverpool, UK) was applied to the plate; this was the primary sensitivity test. With each batch of specimens processed in this way a control plate of DST agar was flood-seeded with a sensitive Escherichia coli NCTC 10418 strain (about $10^{6}$ colony-forming units $(\mathrm{cfu} / \mathrm{ml})$ ) and allowed to dry, and a combined disk containing $1 \mu \mathrm{g}$ trimethoprim and $20 \mu \mathrm{g}$ sulphamethoxazole was applied to the agar surface. The test and control plates were read after overnight incubation at $37^{\circ} \mathrm{C}$. The organisms were classified as sensitive if they gave a zone of sensitivity around the disk equal to or greater than that found in the 
Table 1 Organisms isolated in significant numbers $\left(>10^{8} / l\right)$ from 507 urine specimens

\begin{tabular}{lc}
\hline Organism & Number isolated \\
\hline Escherichia coli & 375 \\
Proteus spp. & 48 \\
Staphylococcus saprophyticus & 39 \\
Klebsiella spp. & 19 \\
Staphylococcus epidermidis & 9 \\
Streptococcus faecalis & 7 \\
Pseudomonas aeruginosa & 5 \\
Staphylococcus aureus & 3 \\
B-haemolytic streptococcus & 2 \\
& Total \\
\hline
\end{tabular}

control, ${ }^{6}$ otherwise the organisms were classified as resistant.

The viable organisms isolated in significant numbers, $>10^{8} \mathrm{cfu} / 1$ ( $\left.>10^{5} \mathrm{cfu} / \mathrm{ml}\right)$, were subcultured on to MacConkey agar, and their identities were checked. This subculture was used to prepare an overnight stationary phase culture in nutrient Broth No. 2 (Oxoid), which was then diluted 1 in $10^{4}$ in Davis-Mingioli basal medium ${ }^{7}$ and flood-seeded on to the surface of Wellcotest Sensitivity Test Agar (Wellcome Laboratories, Beckenham, UK) and allowed to dry. One disk containing trimethoprim $(1 \mu \mathrm{g})$ and another containing sulphamethoxazole $(50 \mu \mathrm{g})$ were placed on the surface of the agar with their centres $25 \mathrm{~mm}$ apart. In addition, a combined disk containing the drugs at these concentrations was also placed on the agar surface. Disks containing $50 \mu \mathrm{g}$ sulphamethoxazole were chosen to take account of the selective antagonism of this antimicrobial drug in thymidine-free medium. ${ }^{4}$ At this concentration the effective trimethoprim-sulphamethoxazole ratio is $1: 20 .{ }^{4}$ Organisms were classified as sensitive if the zone of inhibition was $10 \mathrm{~mm}$ or greater. The drug combination was classified as synergistic according to the criteria described previously. 4

\section{Results}

The types of organism isolated in significant numbers from midstream urine samples from 1 July 1978 to 30 September 1978 are shown in Table 1.

Of the 507 organisms tested, $435(86 \%)$ were $\overline{\bar{c}}$ classified as sensitive to the combined disk used in $\overparen{\nabla}$ the primary sensitivity test (Table 2 ). However, the results show that the true sensitivity patterns of these $\mathrm{c}$ organisms varied considerably. Of the 346 E. coli $\vec{O}$ isolates, $180(52 \%)$ found to be sensitive to the combined disk on primary testing were sensitive to $\vec{\omega}$ both drugs and synergy could be demonstrated; $111(32 \%)$ were sensitive to only one of the two drugs, although synergy could still be demonstrated; $\stackrel{\omega}{\perp}$ and $55(16 \%)$ were either resistant to both drugs but showed demonstrable synergy, or were resistant to $\vec{\oplus}$ one drug without demonstrable synergy. Of the 33 Proteus spp. isolated, $15(45 \%)$ were sensitive to only one of the drugs but synergy was demonstrable, $\vec{T}$ and $3(9 \%)$ were resistant to both drugs although synergy could be demonstrated.

Of the $\mathbf{4 3 5}$ organisms judged to be sensitive in the primary sensitivity test, $229(53 \%)$ were found on secondary testing to be fully sensitive to both drugs with concomitant demonstration of synergy; 140 . $(32 \%)$ were sensitive to one of the drugs and synergy was demonstrated, and $66(15 \%)$ were either resistant to one drug with no demonstrable synergy or were resistant to both drugs but with demonstrable synergy.

On primary sensitivity testing, 72 of the 507 isolates $(14 \%)$ were shown to be resistant to the combined disk. The results (Table 3 ) show that there was some variation in resistance patterns. Of the $29 E$. coli isolates shown to be resistant by primary sensitivity testing $22(76 \%)$ were resistant to both drugs and synergy could not be demonstrated. However, $5(17 \%)$ of these 29 isolates were sensitive to one drug of the combination and

Table 2 Results of secondary double disk sensitivity testing of strains adjudged sensitive to co-trimoxazole on primary single disk testing

\begin{tabular}{|c|c|c|c|c|c|c|c|}
\hline \multirow[t]{2}{*}{ Organism (No. of strains) } & \multicolumn{7}{|c|}{ No. of strains with sensitivity graded as: } \\
\hline & $\begin{array}{l}T^{s} S^{s} \\
+S y n\end{array}$ & $\begin{array}{l}T^{b} S^{r} \\
+S y n\end{array}$ & $\begin{array}{l}T^{r} S^{s} \\
+S y n\end{array}$ & $\begin{array}{l}T^{r} S^{r} \\
+S y n\end{array}$ & $T^{s} S^{r}$ & $T^{r} S^{s}$ & $T^{r} S^{r}$ \\
\hline $\begin{array}{l}\text { Escherichia coli (346) } \\
\text { Proteus spp. (33) } \\
\text { Klebsiella spp. (1) } \\
\text { Staphylococcus saprophyticus (39) } \\
\text { Staphylococcus epidermidis (8) } \\
\text { Staphylococcus aureus (3) } \\
\text { Streptococcus faecalis (5) }\end{array}$ & $\begin{array}{r}180 \\
15 \\
0 \\
26 \\
6 \\
2 \\
0\end{array}$ & $\begin{array}{r}104 \\
6 \\
0 \\
10 \\
2 \\
0 \\
2\end{array}$ & $\begin{array}{l}7 \\
9 \\
0 \\
0 \\
0 \\
0 \\
0\end{array}$ & $\begin{array}{r}12 \\
3 \\
1 \\
0 \\
0 \\
0 \\
1\end{array}$ & $\begin{array}{r}39 \\
0 \\
0 \\
2 \\
0 \\
0 \\
2\end{array}$ & $\begin{array}{l}4 \\
0 \\
0 \\
1 \\
0 \\
1 \\
0\end{array}$ & $\begin{array}{l}0 \\
0 \\
0 \\
0 \\
0 \\
0 \\
0\end{array}$ \\
\hline Total (435) & 229 & 124 & 16 & 17 & 43 & 6 & 0 \\
\hline
\end{tabular}

$\mathrm{T}^{\boldsymbol{s}}=$ trimethoprim sensitive $; \mathrm{T}^{r}=$ trimethoprim resistant $; \mathbf{S}^{s}=$ sulphamethoxazole sensitive $; \mathrm{S}^{r}=$ sulphamethoxazole resistant

+ Syn = synergy demonstrated in vitro. 
Table 3 Results of secondary double disk sensitivity testing of strains adjudged resistant to co-trimoxazole on primary single disk testing

\begin{tabular}{|c|c|c|c|c|c|c|c|}
\hline \multirow[t]{2}{*}{ Organism (No. of isolates) } & \multicolumn{7}{|c|}{ No. of isolates with sensitivity graded as: } \\
\hline & $\begin{array}{l}T^{s} S^{s} \\
+S y n\end{array}$ & $\begin{array}{l}T^{s} S^{r} \\
+S y n\end{array}$ & $\begin{array}{l}T^{r} S^{s} \\
+S y n\end{array}$ & $\begin{array}{l}T^{r} S^{r} \\
+S_{y n}\end{array}$ & $T^{s} S^{r}$ & $T^{r} S^{s}$ & $T^{r} S^{r}$ \\
\hline Escherichia coli (29) & 0 & 3 & 2 & 0 & 2 & $\mathbf{0}$ & 22 \\
\hline Proteus spp. (15) & 3 & 1 & 0 & $\mathbf{0}$ & $\mathbf{0}$ & 0 & 11 \\
\hline Klebsiella spp. (18) & 1 & 3 & 0 & 0 & 0 & 0 & 14 \\
\hline Pseudomonas aeruginosa $(5)$ & 0 & 0 & 0 & 0 & 0 & 0 & 5 \\
\hline Staphylococcus epidermidis (1) & $\mathbf{0}$ & 0 & 0 & 0 & 0 & 0 & 1 \\
\hline$\beta$-haemolytic streptococcus (2) & $\mathbf{0}$ & $1 *$ & 0 & 0 & 0 & 0 & 1 \\
\hline Streptococcus faecalis (2) & 1 & 0 & 0 & 0 & 0 & 0 & 1 \\
\hline Total (72) & 5 & 8 & 2 & 0 & 2 & $\mathbf{0}$ & 55 \\
\hline
\end{tabular}

$\mathbf{T}^{s}=$ trimethoprim sensitive; $\mathbf{T}^{r}=$ trimethoprim resistant $; \mathbf{S}^{s}=\mathrm{s}$ ulphamethoxazole sensitive $; \mathbf{S}^{r}=$ sulphamethoxazole resistant $;$

+ Syn = synergy demonstrated in vitro.

* Primary testing performed on blood agar.

synergy was demonstrated. Of the 15 Proteus spp. found to be resistant on primary testing, $11(73 \%)$ were resistant to both drugs and synergy was not found, but four were sensitive to one or both drugs and synergy could be shown. The Klebsiella spp. isolated showed a very similar pattern, 4 of the 15 apparently resistant strains being sensitive to at least one drug and synergy being present.

Of the 72 strains that were resistant in the primary sensitivity test $55(76 \%)$ were resistant to both drugs and synergy was not shown; $2(3 \%)$ were resistant to one drug and synergy was not shown; and 15 $(21 \%)$ were sensitive to at least one drug and synergy could be demonstrated.

The standard inoculum of each isolate was also examined with a single disk containing $1 \mu \mathrm{g}$ trimethoprim and $50 \mu \mathrm{g}$ sulphamethoxazole. Only those organisms resistant to both components and their combined synergistic action were not susceptible to this disk. Of the 452 organisms that were sensitive to this disk $68(15 \%)$ were either resistant to both components but showed synergy, or were sensitive to one component in the absence of demonstrable synergy. Of these 452 strains $41(9 \%)$ were resistant to trimethoprim.

\section{Discussion}

The comparison of different methods for assessing the sensitivity of bacteria to co-trimoxazole or its components is complicated by the lack of agreement about the criteria that should be used to indicate 'sensitivity'. There is much controversy as to whether the synergy between trimethoprim and sulphamethoxazole, which can be demonstrated easily in vitro, is important in vivo. ${ }^{8}$

Indeed, there is a growing belief that the efficacy of co-trimoxazole often originates from the trimethoprim component alone and that, as long as the organism is trimethoprim-sensitive, the combin2tion may be given regardless of whether synergy or sulphamethoxazole sensitivity can be demonstrated. On these criteria, $396(91 \%)$ of the 435 strains shown to be sensitive by primary testing (Table 2) were sensitive to trimethoprim. In other words, $9 \%$ of the strains that were classified as sensitive as a result of the primary sensitivity test were not in fact sensitive to trimethoprim. More specifically, on these criteria $7 \%$ of E. coli strains, and as many as $36 \%$ of Proteus sp., would have been inappropriately reported after primary combined disk testing.

Of the 72 organisms shown to be resistant by primary sensitivity testing $57(79 \%)$ were found to be resistant to trimethoprim (Table 3) and $15(21 \%)$ would have been inappropriately reported as 'resistant' on primary testing if trimethoprim sensitivity is the criterion for drug administration. Overall, by this criterion, $15(3 \%)$ of the 507 organisms tested were incorrectly reported to be resistant to co-trimoxazole by primary testing. A further 17 organisms $(3 \%)$, which were resistant to both trimethoprim and sulphamethoxazole on secondary testing, were reported as sensitive on primary testing because of in vitro synergy.

If synergy is important, co-trimoxazole therapy might be considered if an organism is sensitive to at least one of the components and in vitro synergy can be demonstrated.

Of the 435 organisms shown to be sensitive in the primary sensitivity test $369(85 \%)$ were sensitive to at least one drug with demonstrable synergy; however, $49(11 \%)$ were sensitive to only one drug and synergy could not be shown. The latter organisms, although shown to be sensitive by the single disk test, would not be regarded as suitable for cotrimoxazole therapy by the above criterion.

Similarly, $57(79 \%)$ of the 72 organisms shown to be resistant in the primary sensitivity test were either 
resistant to both drugs or resistant to one drug without synergy. Thus $21 \%$ of the organisms shown to be resistant would have been regarded as susceptible to co-trimoxazole by the above criterion. Inappropriate sensitivities by primary testing were less of a problem with Klebsiella spp. and Proteus spp.

Finally, it might be argued that an organism should be regarded as sensitive to co-trimoxazole if it is sensitive to either component, regardless of whether or not in vitro synergy is demonstrable. By this criterion no organisms would have been falsely reported as sensitive by the primary combined disk test, but $3 \%$ of the organisms tested were wrongly judged to be resistant.

The results show that not only is a standardised inoculum of paramount importance, but so is the use of separate disks in co-trimoxazole sensitivity testing. In addition, the use of a combined disk in a secondary sensitivity test has no advantage over the use of a similar disk in a primary sensitivity test. The advantages of speed and convenience, which primary combined disk testing has over secondary methods with two disks and a standardised inoculum, must be carefully weighed against the risk of obtaining misleading results or of assuming sensitivity to the combination of trimethoprim and sulphamethoxazole when the organism being tested may be sensitive to only one agent or to neither. At the expense of some delay, which in urinary tract infections may be acceptable, the double disk technique provides useful additional information on the sensitivities of individual organisms to the component drugs.

Although sulphonamides are still used for the treatment of urinary tract infections, ${ }^{9}$ their action is bacteriostatic. Trimethoprim, on the other hand, can be shown to exert a bactericidal effect in vitro at concentrations that can be readily achieved in urine. ${ }^{10}$ If an organism is resistant to sulphamethoxazole, or if synergy should not be important in vivo, then clearly treatment with a redundant agent, which is not free from serious side effects, is undesirable. In such circumstances, treatment with an alternative agent, or with trimethoprim alone, seems more rational.
Whatever viewpoint is taken, the double disk $\stackrel{0}{5}$ technique offers to the microbiologist additional information upon which appropriate treatment can $\overrightarrow{\vec{s}}$ be given.

We thank Catherine McMillan, Gordon Fleming, and Susan McDonald for their assistance. We also $\overrightarrow{\widetilde{d}}$ thank the Scottish Home and Health Department $\varrho$ for their financial support of some of this work is (grant no. K/MRS/50/C163).

\section{References}

${ }^{1}$ Grey D, Hamilton-Miller JMT, Brumfitt W. Combined $\underset{\perp}{\oplus}$ action of sulphamethoxazole and trimethoprim against iv clinically-isolated sulphonamide-resistant bacteria.
Chemotherapy $1979 ; 25: 296-302$.

${ }^{2}$ Acar JF, Goldstein F, Chabbert YA. Synergistic activity 음 of trimethoprim-sulphamethoxazole on Gram-negative bacilli: observations in vitro and in vivo. $J$ Infect Dis $\rightarrow$ $1973 ; 128$ (Suppl): $470-7$.

${ }^{3}$ Anderson JD, Lacey RW, Lewis EL, Sellin MA. Failure to demonstrate an advantage in combining sulphamethoxazole with trimethoprim in an experimental model of urinary infection. J Clin Pathol 1974;27:619-22.

+ Amyes SGB. Synergy and co-trimoxazole sensitivity. $\vec{c}$ $J$ Pharm Pharmacol 1978;30:13P.

${ }^{5}$ Duerden BI, Moyes A. Comparison of laboratory methods in the diagnosis of urinary tract infection. J Clin Pathol 1976;29:286-91.

- Telfer Brunton WA, Heggie D. A study of urinary tract infection in general practice. $J$ Infect 1979;1:183-9.

7 Davis BD, Mingioli ES. Mutants of Escherichia coli requiring methionine or vitamin B12. J Bacteriol 1950; 60:17-28.

${ }^{8}$ Greenwood D, O'Grady FW. Activity and interaction of $\overline{\overline{0}}$ trimethoprim and sulphamethoxazole against Escherichia coli. J Clin Pathol 1976;29:162-6.

${ }^{\circ}$ Källenius G, Winberg J. Urinary tract infections treated with single dose of short-acting sulphonamide. $\mathrm{Br} \mathrm{Med}$ $J$ 1979; ;:1175-6.

${ }^{10}$ Amyes SGB, Smith JT. Trimethoprim action and its analogy with thymine starvation. Antimicrob Agents Chemother 1974;5:169-78.

Requests for reprints to: Dr WA Telfer Brunton, 을 Department of Bacteriology, The Medical School, $D$ University of Edinburgh, Teviot Place, Edinburgh EH8 9AG. 